\title{
Rainfall Simulations of Typhoon Morakot with Controlled Translation Speed Based on EnKF Data Assimilation
}

\author{
Tzu-Hsiung Yen, Chun-Chieh Wu *, and Guo-Yuan Lien \\ Department of Atmospheric Sciences, National Taiwan University, Taipei, Taiwan
}

Received 14 February 2011, accepted 5 July 2011

\begin{abstract}
Typhoon Morakot produced record-breaking accumulated rainfall over southern Taiwan in August 2009. The combination of several factors resulted in this extreme weather event: the steep terrain in Taiwan, the prevailing south-westerly flow in the monsoon trough, Typhoon Goni over the northern South China Sea, and the slow translation speed of Morakot itself over Taiwan. In this study, the influence of the translation speed is particularly emphasized. Based on the EnKF data assimilation, an innovative method is applied to perform ensemble simulations with several designated translation speeds of Morakot using the WRF model. Thus the influence of the translation speed on the amount of accumulated rainfall over Taiwan can be quantitatively evaluated. In the control simulation with observed translation speed, the maximum amount and geographic pattern of accumulated rainfall during the landfall period of Morakot are generally consistent with the observations, though the detailed overall distributions of accumulated rainfall is mostly underestimated, resulting in the low bias of the frequency distribution of the accumulated rainfall. In a simulation with nearly-doubled translation speed of Morakot, the maximum accumulated rainfall is decreased by $33 \%$ than that in the control simulation, while the rainfall distribution over Taiwan remains similar. In addition, the 28 ensemble members can further provide additional information in terms of their spread and other statistics. The results from ensemble members reveal the usefulness of ensemble simulations for the quantitative precipitation forecast.
\end{abstract}

Key words: Rainfall, Typhoon Morakot, Translation speed, EnKF data assimilation, Ensemble simulations

Citation: Yen, T. H., C. C. Wu, and G. Y. Lien, 2011: Rainfall simulations of Typhoon Morakot with controlled translation speed based on EnKF data assimilation. Terr. Atmos. Ocean. Sci., 22, 647-660, doi: 10.3319/TAO.2011.07.05.01(TM)

\section{INTRODUCTION}

Tropical cyclone (TC) is one of the most devastating weather systems, and one of the most important severe weather events in Taiwan. The rainfall associated with TCs is an important component of water resources in Taiwan. Taking year 2009 as an example, cold front systems in spring, Mei-Yu systems, and afternoon thunderstorms during that summer did not bring enough rainfall to Taiwan. As a result, the government and the residents of Taiwan started to restrain water usage because of low water storage levels around Taiwan. Nevertheless, abundant rainfall came unexpectedly with Typhoon Morakot on 6 to 9 August. It not only filled up all water reservoirs in three days but also led to severe mudslides and flooding, resulting in more than 700 casualties and enormous economic damage. To mitigate

\footnotetext{
* Corresponding author

E-mail: cwu@typhoon.as.ntu.edu.tw
}

this damage, improving TC forecasts, including not only the tracks, but also the rainfall amount and distribution, is one of the most urgent and challenging tasks regarding $\mathrm{TC}$ research and forecasting in Taiwan.

The most important characteristic of Morakot is its astoundingly heavy precipitation. Heavy rainfall over southern Taiwan usually occurred in an environment with prevailing moisture-rich south-westerly flow, in which mesoscale disturbances are embedded (Chen and Chen 2003). The steep terrain also plays a key role in substantially increasing the total rainfall amounts over Taiwan (Wu and Kuo 1999; Wu 2001; Wu et al. 2002). There have been a series of papers aiming to address the scientific and forecasting issues related to Morakot after the event. Based on a synoptic analysis and various types of observation data including satellite and radar images, the torrential rainfall in the southwestern Taiwan in three consecutive days was found to be closely related to huge continuously-developing convective 
rainbands over the Taiwan Strait, which can be partly attributed to the convergence of northerly wind associated with TC outer circulation and the south-westerly monsoon flow (Jou et al. 2010; Lin et al. 2010; Wang et al. 2010). From a broader perspective, Hong et al. (2010) showed that Morakot was embedded in a monsoon circulation of different time scales which provided a favorable environment. The record-breaking rainfall was the result primarily from the typhoon vortex and the submonthly large-scale cyclonic disturbance. The influence of the quasi-biweekly-scale gyre and low-frequency monsoon flows on the slow movement and asymmetric precipitation of Morakot was also emphasized in observational (Wu et al. 2011b) and modeling (Liang et al. 2011) studies. In addition, Yeh et al. (2010) reviewed the official forecast provided by the Central Weather Bureau (CWB) during the warning period of Morakot. The 24-h track forecast error of CWB in this case is smaller than the averaged value among all previous TCs, while the overestimated translation speed of Morakot near Taiwan results in the considerably underestimated influence on the island.

On the other hand, high-resolution simulation using mesoscale models, such as the fifth-generation Pennsylvania State University-National Center for Atmospheric Research Mesoscale Model (MM5) and Weather Research and Forecasting (WRF) model, can further improve our understanding of the mechanism of this heavy rainfall event. The rainfall pattern over Taiwan can be reasonably simulated in these models, provided that the model and terrain resolution is sufficient and the TC track in the model doesn't excessively deviate from the observation (Wu et al. 2002, 2010a; Ge et al. 2010; Zhang et al. 2010; Hendricks et al. 2011; Tao et al. 2011). Better model forecasts on the track and intensity of Morakot can be obtained with appropriate implementation of various TC initialization schemes (Huang et al. 2011; Nguyen and Chen 2011). The roles of the terrain and other typhoon, Goni, have been discussed in these studies. The peak value of the accumulated rainfall will decrease significantly and the rainfall pattern will shift eastward if a reduced terrain height over the island of Taiwan is used in the model (Ge et al. 2010; Huang et al. 2011). Typhoon Goni, circling around the northern South China Sea during the same period as Morakot, also played an important role in enhancing the wind speed of the southwesterly flow as well as elevating the moisture content in such flow. Therefore, the heavy rainfall in southern Taiwan was further enhanced (Jou et al. 2010; Huang et al. 2011; Nguyen and Chen 2011).

The slow translation speed of Morakot is also a critical factor leading to the significant accumulated rainfall. The slower a TC moves, the longer it influences Taiwan. As a result, the duration of rainfall lasts longer, and the total accumulated precipitation amounts to a higher level. Lin et al. (2001) pointed out that the slow movement of a convective system is a common ingredient contributing to heavy oro- graphic rainfall. Morakot is a case in point and highlights the importance of translation speed of a landfalling TC. However, the lack of ability to control TC motion in numerical model hinders us from examining this factor more quantitatively with numerical simulations.

At this point, directly assimilating the tracks of a hurricane-like vortex into the model based on the ensemble Kalman Filter (EnKF; Evensen 1994, 2003) is a possible method to address this issue. This method has been tested and examined using a 2-dimentional barotropic model (Chen and Snyder 2007) and a full-physics high-resolution mesoscale model (i.e., WRF model; Torn and Hakim 2009; Wu et al. 2010b). Based on the framework used by Wu et al. (2010b), we design a series of numerical experiments with different TC translation speeds to quantitatively assess its impact on the accumulated rainfall amount associated with Morakot. The innovative method to control TC motion in the mesoscale model is the focus of this short article, and the advantages of ensemble forecast for quantitative precipitation forecast (QPF) are also discussed in this study.

The lifecycle of Typhoon Morakot and its influence on Taiwan are briefly reviewed in section 2 . Section 3 describes the methodology and experimental design. Section 4 shows the results of ensemble simulations and the rainfall characteristics in terms of two different TC translation speeds. Section 5 presents several concluding remarks.

\section{OVERVIEW OF TYPHOON MORAKOT}

In early August 2009, the monsoon trough over the western North Pacific was relatively active. Typhoon Morakot, the dominating feature in this monsoon trough, formed to the east of Taiwan at 0000 UTC 03 August. It then headed steadily westward and made landfall at the central east coast of Taiwan at 1600 UTC 07 August, with a maintained intensity around category 1 to 2 . At the same time, another weaker typhoon, Goni, formed at 1200 UTC 03 August, circled cyclonically over the Hainan Island region. There seems to be a Fujiwhara effect (Lander and Holland 1993; $\mathrm{Wu}$ et al. 2003) between these two typhoons. Goni eventually weakened mostly due to the intense environmental vertical shear over the northern South China Sea. In such a period, Morakot and Goni, associated with a third tropical depression (the predecessor vortex of Typhoon Etau), composed a broader monsoon gyre (Lander 1994) with a zonal span of more than $4000 \mathrm{~km}$ (Fig. 1).

After Morakot made landfall in Taiwan, its translation speed decreased significantly from more than $20 \mathrm{~km} \mathrm{~h}^{-1}$ to around $10 \mathrm{~km} \mathrm{~h}^{-1}$. This fact resulted in a prolonged influence time in Taiwan, and the heavy rainfall thus lasted for more than 4 days. Figure 2 shows the observed 4-day (0000 UTC 06 August - 0000 UTC 10 August) accumulated rainfall from the rain gauge network. The heavy rainfall covered most of the mountain area and south-western plain of Tai- 


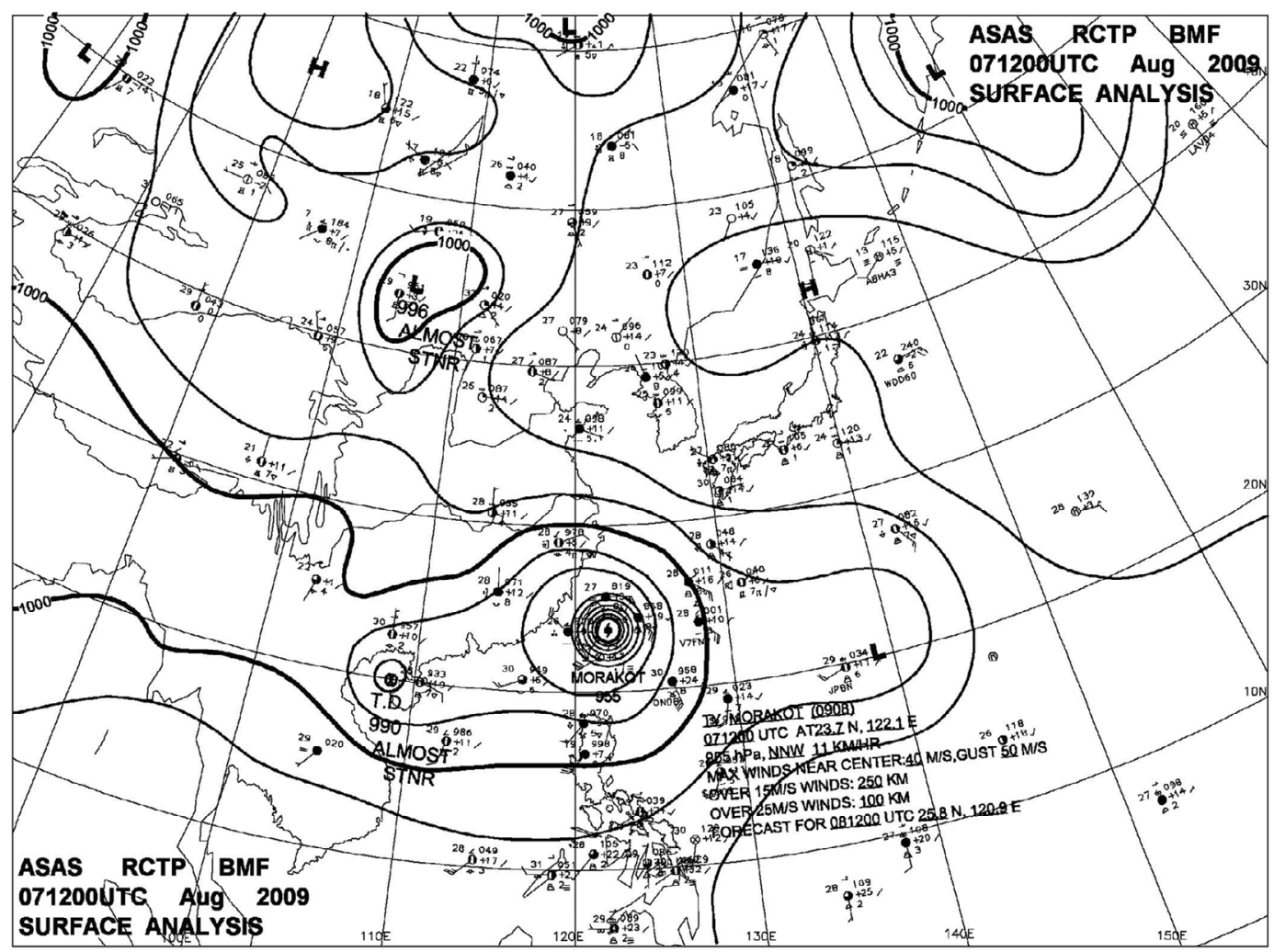

Fig. 1. The surface analysis at 1200 UTC 07 August 2009 by the Central Weather Bureau.

wan, and amounted to a maximum value of around $3000 \mathrm{~mm}$ during these four days on the south-western slope of the Central Mountain Range (CMR) of Taiwan. In the most intense rainfall period selected in this study (1200 UTC 07 August - 1800 UTC 08 August), Morakot produced a maximum accumulated rainfall of $1609 \mathrm{~mm}$ at Wei Liao Shan station within 30 hours (Fig. 6d).

\section{METHODOLOGY}

\subsection{Model Settings and EnKF Data Assimilation System}

The ensemble Kalman filter (EnKF) first proposed by Evensen (1994) is a powerful and efficient method to optimally estimate model state from the background and observations with the flow-dependent background error statistics. In recent years, it has been extensively adopted in atmospheric numerical models for the use of research in various topics (Snyder and Zhang 2003; Torn and Hakim 2009; Zhang et al. 2009) and of operational weather prediction systems (Szunyogh et al. 2008; Torn and Hakim 2008; Whitaker et al. 2008). In addition, it has been shown that the performance of EnKF is generally better than the 3-dimensional variational (3D-VAR) data assimilation scheme and is comparable to the 4-dimensional variation (4D-VAR) method (Anderson et al. 2009). Wu et al. (2010b) used the ensemble square root filter (EnSRF; Whitaker and Hamill

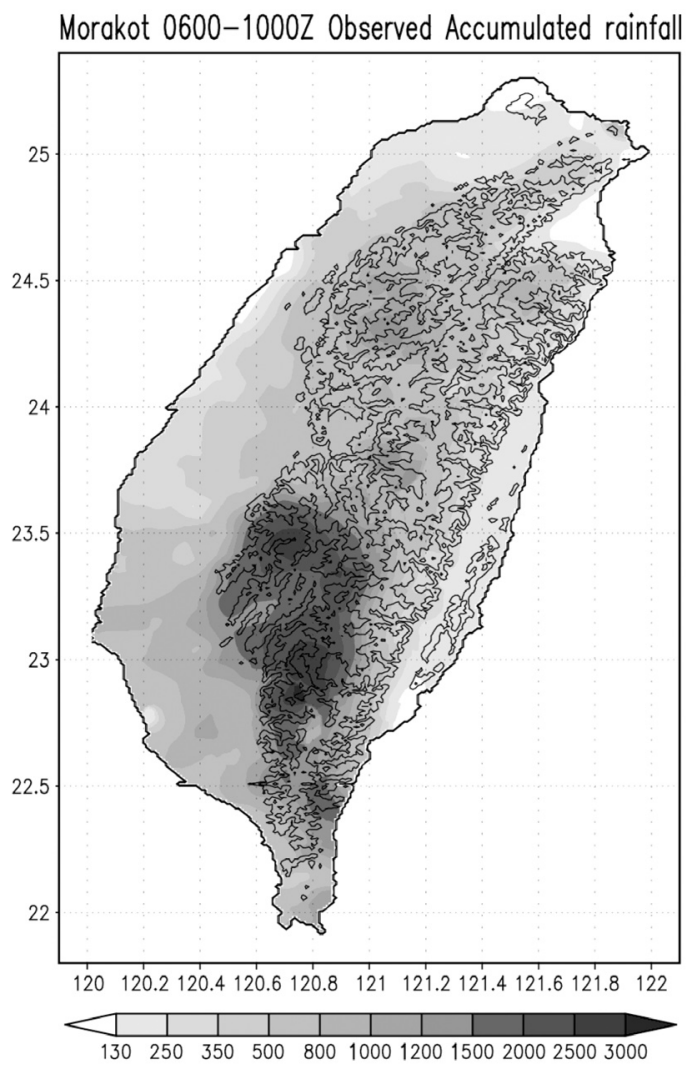

Fig. 2. The observed 4-day accumulated rainfall (mm) of Morakot from 0000 UTC 06 August to 0000 UTC 10 August. 
2002) to assimilate TC center position, storm motion vector, and axisymmetric surface wind profile directly into the model, by constructing these three newly proposed observation operators for TCs. They employed this method to initialize a high-resolution inner-core structure of Typhoon Fung-wong (2008). In addition, a cycling run has been performed to obtain a reasonable analysis field of Typhoon Sinlaku (2008) spanning the whole eyewall replacement cycle (Wu et al. 2011a). The same WRF-based EnKF data assimilation system is used in this study. Detailed discussions of this method are presented in Wu et al. (2010b).

Figure 3 shows the domain settings of the Advanced Research WRF (ARW) model (version 2.2.1). The horizontal grid spacing is $30 \mathrm{~km}(151 \times 121$ grid points $), 10 \mathrm{~km}$ $(166 \times 130$ grid points $)$ and $3.33 \mathrm{~km}(82 \times 127$ grid points $)$ for the $1^{\text {st }}(\mathrm{D} 1), 2^{\text {nd }}(\mathrm{D} 2)$ and $3^{\text {rd }}(\mathrm{D} 3)$ domain, respectively. Unlike the vortex-following inner domain used in $\mathrm{Wu}$ et al. (2010b, 2011a), all domains are fixed in this simulation. Restricted by the computational resources available, only D1 and D2 are used during the ensemble integration and data assimilation. The $10-\mathrm{km}$ resolution of D2 is acceptable to resolve the broad and loose inner-core structure of Morakot when it is over the ocean. The additional D3, which is only presented in the ensemble mean, one-way feedback from its parent domain (D2), is exactly located in the Taiwan terrain to better resolve the terrain-induced precipitation. There are 35 vertical levels in the terrain-following sigma coordinate (same as those in Wu et al. 2010b). The physics parameterization schemes used in the simulations is WRF Single Moment (WSM) 6-class graupel microphysics scheme (Hong et al. 2004; Hong and Lim 2006), Rapid Radiative Transfer Model (RRTM) scheme (Mlawer et al. 1997) for long-wave radiation, the simple short wave scheme (Dudhia 1989) for short-wave radiation, and the Yonsei University (YSU) planetary boundary layer scheme (Hong et al. 2006). The cumulus convection is parameterized with the Grell-Dévényi ensemble scheme (Grell and Dévényi 2002) only in D1 and D2.

Taking the National Centers for Environmental Prediction (NCEP) final analysis $\left(\mathrm{FNL} ; 1^{\circ} \times 1^{\circ}\right)$ at $0000 \mathrm{UTC} 06$ August 2009 as the initial ensemble mean, the 28 ensemble members are generated by randomly perturbing the mean analysis in a transformed streamfunction field as described in Zhang et al. (2006). This method guarantees that the horizontal wind, temperature, and pressure of the initial perturbation field are in geostrophic balance. After a pre-run in the first 3 hours, the TC center position, storm motion vector, and axisymmetric surface wind profile are assimilated into the model with an update cycle of every hour to initialize a reasonable vortex structure of Typhoon Morakot. The hourly observations of TC center position and storm motion vector are computed from the JTWC best-track data by means of the cubic spline interpolation. The axisymmetric surface wind profile is obtained based on Willoughby's empirical formula (Willoughby et al. 2006). The given observation errors of each observation are similar to those in $\mathrm{Wu}$ et al (2010b). Besides, the covariance relaxation parameter $(\alpha$; Zhang et al. 2004) is set at 0.85 and the influence radius of the covariance localization is set at $800 \mathrm{~km}$ also based on previous tests (Wu et al. 2010b). The assimilation of axisymmetric surface wind profile is suspended at 1200 UTC 07 August when its circulation is very close to the Taiwan terrain, because the interaction between Morakot and the Taiwan terrain should result in a highly-asymmetric surface wind structure and the surface friction also reduces the $10-\mathrm{m}$ surface wind considerably in the model. This situation does not meet the original idea of designing this special TC observational parameter. While the conventional observation data could be included in the assimilation to improve the analysis of environmental field and lead to better TC simulations (Wu et al. 2011a), they are not assimilated into the model in this study. As stated above, the 3-day simulation from 0000 UTC 06 August to 0000 UTC 09 August is regarded as the control (CTRL) experiment.

\subsection{Controlled TC Track Based on EnKF}

In addition to the CTRL experiment, to quantitatively evaluate the impact on accumulated rainfall associated with the translation speed of Morakot, other experiments, FAST and SLOW, are initiated at 1200 UTC 07 August with hypothetical faster and slower tracks, respectively. The track of FAST is artificially constructed by skipping every two center position data after 1200 UTC 07 August, i.e., the center position at 1800 UTC 07 August is replaced by that originally at 0000 UTC 08 August, and the center position at 0000 UTC 08 August is replaced by that originally at 1200 UTC 08 August, and so on. On the other hand, the track of SLOW

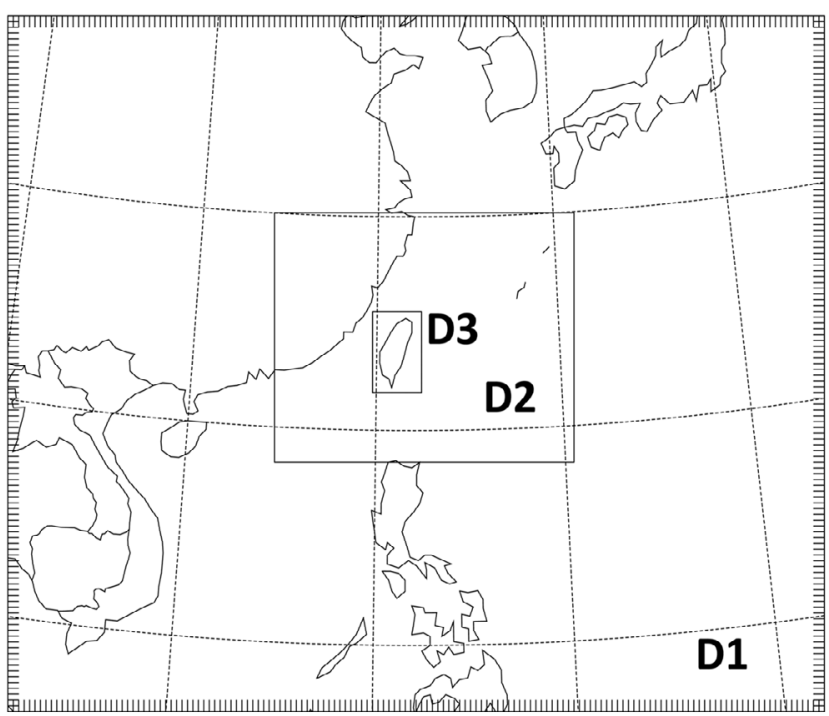

Fig. 3. Three domains of the WRF model. 
is constructed by assigning the delayed time to each center position data so that the translation speed is halved after 1200 UTC 07 August. Then, the hypothetical fast-moving or slow-moving tracks are assimilated into the model based on the aforementioned technique in order to force the simulated Morakot move with doubled or halved speed than that of observation. In the following sections of this study, only the results from CTRL and FAST (not SLOW) experiments will be analyzed and compared because the TC translation speed in SLOW is unrealistic slow while that in FAST is, in contrast, falling within the common value of landfalling TCs in Taiwan.

\section{RESULTS}

\subsection{The Resulting Tracks of Each Experiment}

As shown in Fig. 4, the ensemble mean track of Morakot in CTRL (long dashed line) matches the observation (solid line with TC marks) well (root mean square TC position error of about $49.7 \mathrm{~km}$ through the whole data assimilation period of 3 days), thus indicating that the method can effectively assimilate the observed track of Morakot. The effectiveness of assimilating the TC track and mean structure by the EnKF method has also been demonstrated by $\mathrm{Wu}$ et al. (2010b, 2011a). However, overall, the minimum central sea surface pressure of the simulated Morakot is higher than the JTWC estimation by about 10 to $20 \mathrm{hPa}$ (Fig. 5), possibly because the mean structure has not been assimilated after 0000 UTC 07 August as the storm is about to make landfall. Therefore, the intensity is not well represented during the landfall period. Meanwhile, in the FAST experiment, the artificial constraint on the storm movement results in a fast-moving TC after 1200 UTC 07 August 2009 (short dashed line in Fig. 4). Although there are few variations during the landfall period, the overall TC translation speed in FAST after 1200 UTC 07 August is about twice as fast as the observation (Fig. 4), which is consistent with the original purpose of the EnKF data assimilation. Note that through the rapid update cycle of TC center and motion vector assimilation, the evolution of those analyses or shortterm (1 hour) model forecasts does not only come from the model dynamics, but also the artificial modification introduced in each analysis step. As a result, the Morakot simulation can move much faster than the observation.

\subsection{Comparison of Accumulated Rainfalls}

The accumulated rainfall amounts in the hourly updatecycle simulations are computed with the summation of the rainfall amounts in each 1-hour forecast. To more precisely compare the results between each experiment with different $\mathrm{TC}$ translation speeds, all the following comparisons of their

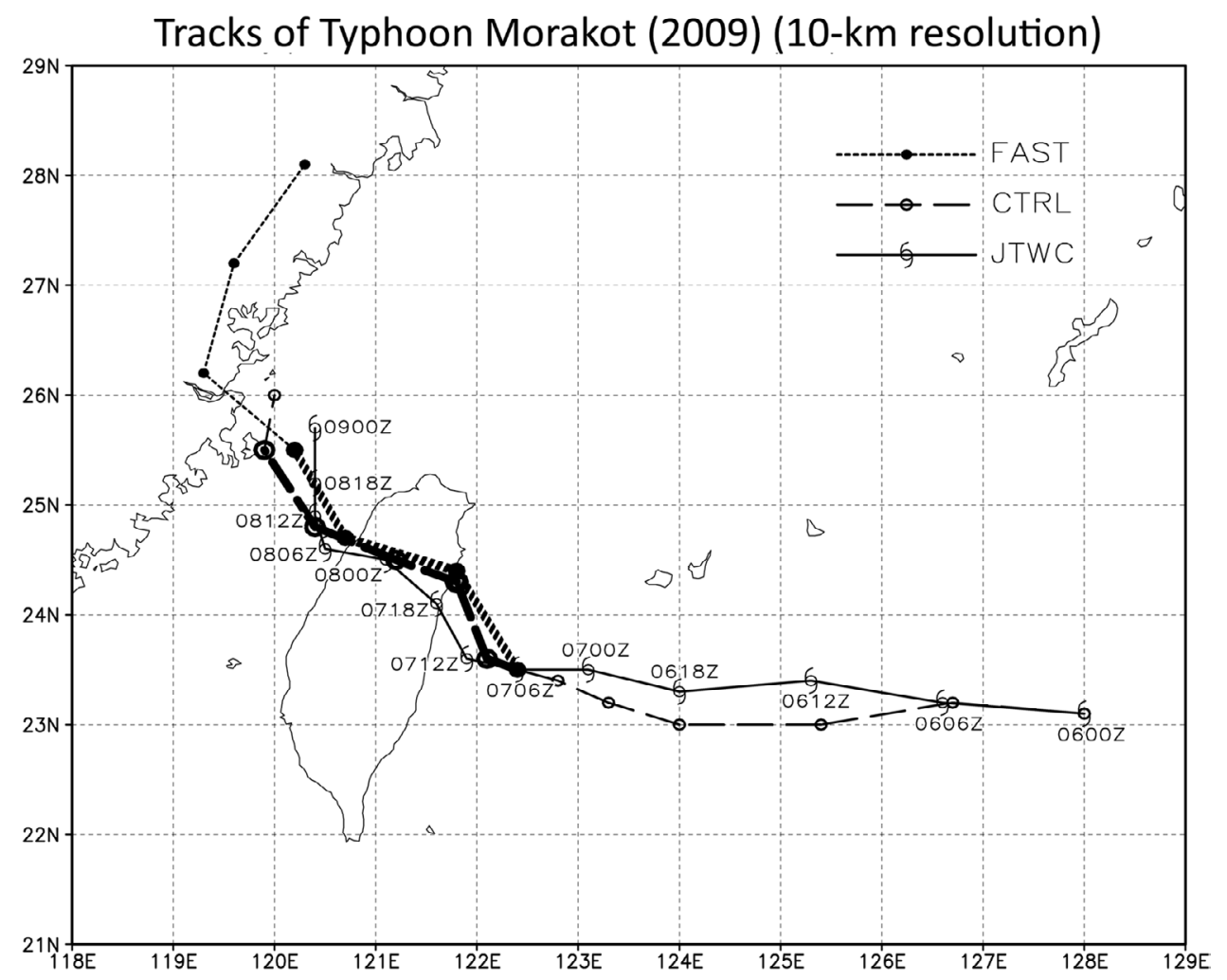

Fig. 4. The ensemble mean track of Morakot in CTRL (long dashed line) and FAST (short dashed line), and the observed track from JTWC (solid line with TC marks). The intervals chosen to make a comparison of accumulated rainfall are highlighted by enhanced thick lines. 


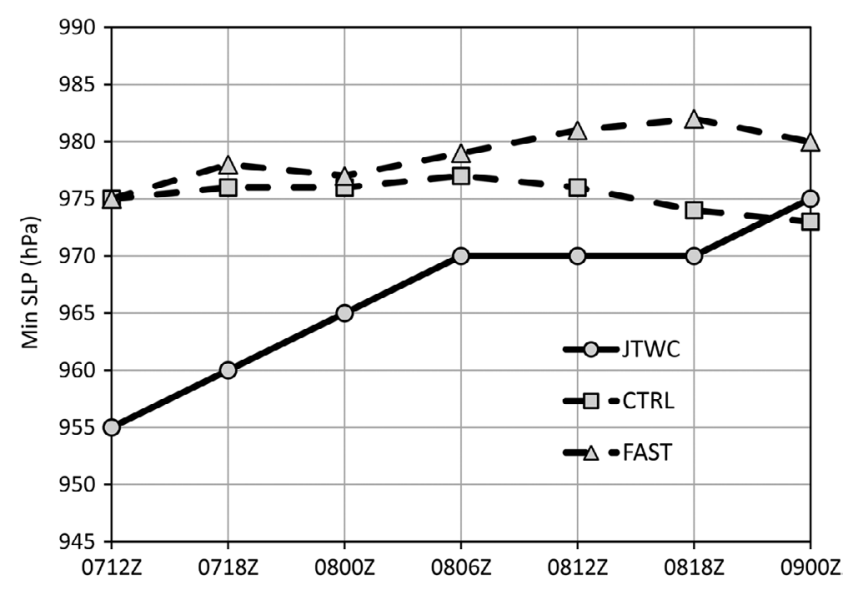

Fig. 5. The minimum sea level pressure (hPa) of Morakot in CTRL (dashed line with square marks), FAST (dashed line with triangle marks), and JTWC estimation (solid line) during the landfall period.

accumulated rainfall are made based on the time intervals in which the simulated TCs pass through the similar section of track, namely, the whole landfall period in addition to roughly $100-\mathrm{km}$ passage over the ocean at the beginning and end (Fig. 4). The corresponding times are 1200 UTC 07 August to 1800 UTC 08 August (30 hours; enhanced thick long dashed line segment in Fig. 4) in CTRL, 1200 UTC 07 August to 0600 UTC 08 August (18 hours; enhanced thick short dashed line segment in Fig. 4) in FAST, and 1200 UTC 07 August to 0000 UTC 10 August (60 hours; not shown) in SLOW, respectively. Note that although the selected periods are different in order to match up the movements of TCs, the lack of the latter 12-h interval in FAST has no significant influence on the accumulated rainfall amount (figure not shown). It is because the Morakot simulation in FAST has moved far away from the island of Taiwan and has made landfall in China (Fig. 4). The sequential 6-hourly-averaged translation speeds of Morakot within the periods for comparison are listed in Table 1. The averaged translation speed during this period of JTWC, CTRL, FAST, and SLOW are $8.7,12.0,18.6$, and $6.2 \mathrm{~km} \mathrm{~h}^{-1}$, respectively.

The simulated rainfall amounts in D3 with $3.33-\mathrm{km}$ resolution (ensemble mean) are shown in Fig. 6. Compared with the observation from the rain gauge network in Taiwan (Fig. 6d), CTRL, FAST and SLOW all capture a similar rainfall pattern: heavy rainfall along windward sloping areas in southern Taiwan. The maximum rainfall in CTRL is $1800 \mathrm{~mm}$, which is close to the observed amount of 1609 $\mathrm{mm}$, during the same 30-h period (Table 1). A number of recent simulations on Morakot based on WRF or MM5 model (Ge et al. 2010; Lin et al. 2010; Zhang et al. 2010; Huang et al. 2011; Nguyen and Chen 2011; Tao et al. 2011) also showed that these mesoscale models have the ability to produce extreme rainfall amounts comparable to the observation. However, it is found that coverage of the low- to medium-rainfall area in the simulation is narrower than the observation, especially in the south-western plain and central-northern mountain area in Taiwan. The extreme rainfall is thus restricted to the north-south elongated area located at the southern CMR and slightly southward to where it was observed. This rainfall characteristic can also be found in some independent studies using the WRF model with similar experimental settings and resolution (Lin et al. 2010; Huang et al. 2011; Tao et al. 2011). Compared with the observed development of convective patterns during the landfall period of Morakot, all of these simulations may fail to simulate the rainband located between the northwesterly flow associated with the Morakot circulations and the southwesterly monsoon flow. In the simulation by Nguyen and Chen (2011), the refinement of the model initial condition via the advanced vortex initialization technique would substantially improve the results, in which the asymmetric structure of the essential rainband was successfully simulated. Nevertheless, the causes of this problem remain unclear at this stage and are worthy of further examination.

Although the rainfall pattern remains the same in FAST, the maximum value only reaches $1207 \mathrm{~mm}$ within the corresponding period (Table 1), reflecting the influence of accumulated rainfall brought about by the translation speed of Morakot. The difference in accumulated rainfall amount between these two experiments (CTRL minus FAST) is also shown in Fig. 6e. Clearly, different translation speeds of TCs lead to a significant difference in the accumulated rainfall over Taiwan. In all, a 55\% increase (from 12.0 to $18.6 \mathrm{~km} \mathrm{~h}^{-1}$ ) of the translation speed of Typhoon Morakot leads to a 33\% reduction (from 1800 to $1207 \mathrm{~mm}$ ) in the maximum accumulated rainfall over Taiwan. In addition, the accumulated rainfall in SLOW can further verify the important role of translation speed of TCs in the extreme rainfall event. The maximum accumulated rainfall amount reaches $2259 \mathrm{~mm}$ when the simulated TC passes Taiwan at a fairly slow pace (Fig. 6c). It is worth pointing out that in addition to the increasing maximum rainfall amount, the low- to medium-rainfall area is notably broadened in SLOW, especially around the two secondary local maxima in the central-western (Ali Shan) and north-western (Hsueh Shan) mountain areas. The overall rainfall pattern turns out to be more similar to the observation though the translation speed of simulated TC in SLOW is less than the observed. This result is likely attributed to the enhanced accumulated effect due to the longer influence time of Morakot over Taiwan. Meanwhile, it is worth noting that the model physics used in this study is able to depict the rainfall mechanism and reproduce the precipitation pattern although the total rainfall amount may not be in accord with the observation.

\subsection{Ensemble Spread in Rainfall Simulations}

The value of the ensemble is not limited to performing 
Table 1. The periods compared for accumulated rainfall of observation, CTRL, FAST, and SLOW experiments. During these periods, the previous 6-hourly and the overall averaged translation speeds of Morakot $\left(\mathrm{km} \mathrm{h}^{-1}\right)$ are listed (the translation speed of the last five 6-hour intervals in SLOW are shown by their average value), in addition to the maximum rainfall amounts of observation and in D3 of each experiment (mm).

\begin{tabular}{|c|c|c|c|c|c|c|c|c|c|}
\hline & \multirow{3}{*}{$\begin{array}{c}\text { Periods } \\
\text { compared }\end{array}$} & \multicolumn{7}{|c|}{ Translation speed $\left(\mathrm{km} \mathrm{h}^{-1}\right)$} & \multirow{3}{*}{$\begin{array}{l}\text { Maximum } \\
\text { rainfall in D3 } \\
\quad(\mathrm{mm})\end{array}$} \\
\hline & & \multicolumn{6}{|c|}{ Previous 6 hours } & \multirow[b]{2}{*}{ Average } & \\
\hline & & $0718 Z$ & $0800 \mathrm{Z}$ & $0806 \mathrm{Z}$ & $0812 Z$ & $0818 Z$ & $\begin{array}{c}0900-1000 \mathrm{Z} \\
\text { (average) }\end{array}$ & & \\
\hline Observation & $0712 \sim 0818 \mathrm{Z}$ & 10.6 & 11.2 & 10.3 & 5.8 & 5.6 & & 8.7 & 1609 \\
\hline CTRL & $0712 \sim 0818 \mathrm{Z}$ & 5.4 & 13.9 & 10.8 & 14.6 & 15.4 & & 12.0 & 1800 \\
\hline FAST & $0712 \sim 0806 \mathrm{Z}$ & 19.5 & 19.3 & 17.0 & & & & 18.6 & 1207 \\
\hline SLOW & $0712 \sim 1000 Z$ & 3.9 & 1.9 & 7.4 & 7.6 & 13.6 & 5.6 & 6.2 & 2259 \\
\hline
\end{tabular}

(a) CTRL Max: $1800 \mathrm{~mm}$

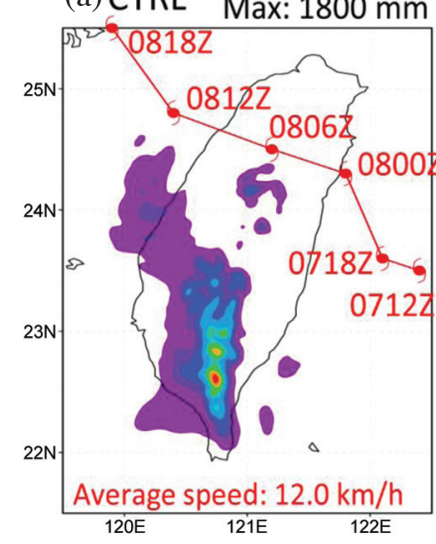

(b) FAST

Max: $1207 \mathrm{~mm}$

(c) SLOW

Max: $2259 \mathrm{~mm}$

(d) OBS

Max: $1609 \mathrm{~mm}$
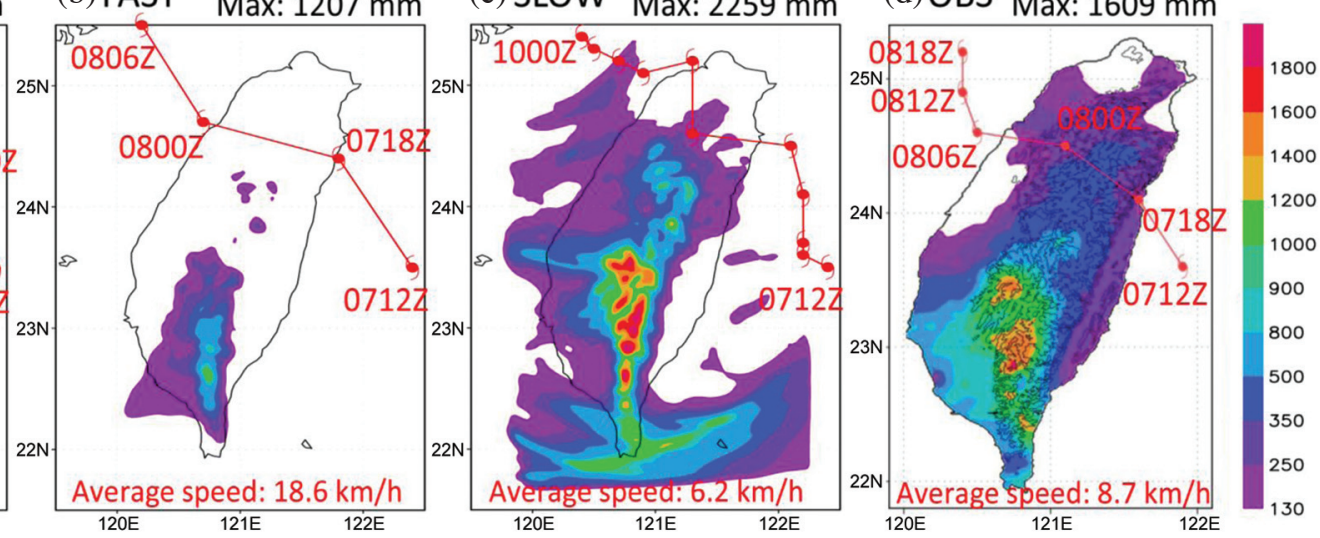

(e) CTRL-FAST

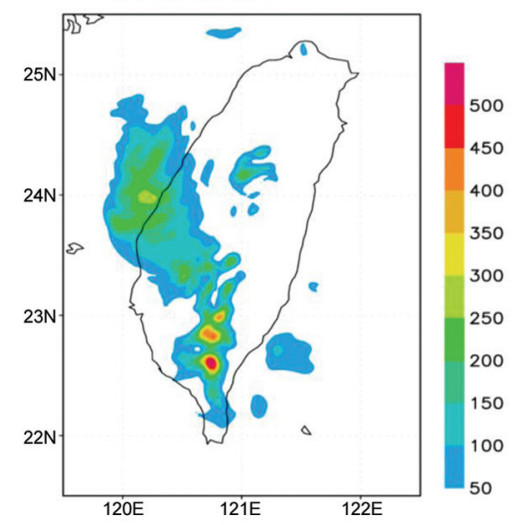

Fig. 6. The accumulated rainfall (mm) (a) from 1200 UTC 07 August to 1800 UTC 08 August in CTRL, (b) from 1200 UTC 07 August to 0600 UTC 08 August in FAST, (c) from 1200 UTC 07 August to 0000 UTC 10 August in SLOW, and (d) from 1200 UTC 07 August to 1800 UTC 08 August by the rain gauge network in Taiwan (mm). The corresponding track segments of Morakot during these periods are also shown as a red line. (e) The difference in accumulated rainfall ( $\mathrm{mm}$ ) between (a) and (b) (CTRL minus FAST). All figures are plotted using domain 3.

the EnKF data assimilation, but it also provides additional information of the spread and other statistics over the ensemble members. The ensemble tracks and ensemble rainfall distributions in CTRL and FAST are shown in Figs. 7 to 9. As mentioned in section 3, only D1 and D2 are used during the ensemble integration and data assimilation because of the limitation in computational resources. Therefore, these figures here are plotted using the finest available domain, D2, with $10-\mathrm{km}$ resolution. It is found that the ensemble TC tracks in both CTRL (Fig. 7a) and FAST (Fig. 7b) keep a certain spread during the landfall period. In some members, the Morakot simulation passes the northern-most tip of Taiwan, while it crosses the main part of CMR in some other members. This dataset provides a unique opportunity to explore the rainfall characteristics with respect to the deviation of TC tracks. The accumulated rainfalls from 1200 UTC 07 August to 1800 UTC 08 August of all ensemble members in CTRL are plotted in Fig. 8. The ensemble member displays some diverse rainfall patterns and amounts. Generally speaking, all members are able to simulate heavy rainfall on 
the windward side of the southern CMR. Nevertheless, the maximum value of the rainfall ranges from $456 \mathrm{~mm}$ (member \#28) to $1081 \mathrm{~mm}$ (member \#20) in spite of minor differences in TC tracks (Fig. 7a). The result of variant rainfall amounts is consistent with Zhang et al. (2010), although they obtained more diverse rainfall patterns as a result of the much larger spread of TC tracks in their free-run ensemble forecasts without data assimilation. This fact highlights the uncertainty and difficulty regarding the QPF of TCs, while implying the potential of the probabilistic forecasts. Note that the reduction of the maximum rainfall here compared with Fig. 6 is mainly due to the coarser resolution of D2. This issue will be discussed in the next subsection. Moreover, even with the coarser $10-\mathrm{km}$ grids, the investigation of rainfall patterns would still be helpful, and some members exhibit a pattern close to that which was observed (e.g., member \#3, \#13, \#19). Regarding the FAST experiment (Fig. 9), the overall characteristics remain the same, while the maximum rainfall amounts are less than those in CTRL, ranging from 315 (member \#28) to 865 (member \#15) mm.

\subsection{Rainfall Distribution with Respect to Taiwan Terrain and Model Resolution}

The cumulative frequency of the total rainfall in D3 based on the data points over the island of Taiwan is calculated and shown in Fig. 10, as well as that for the rain gauge observation. It is clear that the area of medium rainfall in observation is much broader than those areas in both CTRL and FAST simulations as mentioned in the previous subsection. Also, rainfall distribution in FAST is more scattered with smaller accumulated amount than that in CTRL, especially at the high-rainfall end. For example, the area with a rainfall amount higher than $500 \mathrm{~mm}$ in FAST is nearly half the size of that in CTRL. In addition, it is interesting that in SLOW the cumulative frequency is closest to the observation, though there are obviously overestimated accumulative frequencies at both low- and high-rainfall ends. It is consistent with what we found in Fig. 6 where the rainfall pattern in SLOW is much broader than those in CTRL and FAST. Again, this fact highlights the critical role of TC translation speed in the extreme rainfall event.

Furthermore, rainfall distribution with respect to the Taiwan terrain is also presented. All data points over the island are categorized based on their terrain height. The average accumulated rainfall of each 500-m height interval in each domain of each experiment is shown in Fig. 11. For the results from D3 with 3.33-km resolution (solid lines in Fig. 11), the rainfall amount increases with the increase of terrain height from 0 to 1500 meters, and then it is saturated over the high mountain area with about $300-\mathrm{mm}$ average rainfall in CTRL and 220-mm average rainfall in FAST. This distribution is consistent with the results from independent simulations in Tao et al. (2011), which also concluded that most of the surface rainfall occurs over terrain above 1500 meters.

Meanwhile, a significant reduction of the average rainfall is found when the results from D2 are discussed (dashed lines in Fig. 11). This reduction is mainly attributed to the insufficient resolution of model variables, especially the terrain height, which cannot properly resolve the precipitation process associated with the topography-enhanced vertical motion (Wu et al. 2002). As shown in Figs. 12a, b, the insufficient resolution in D2 leads to a poor representation of the Taiwan terrain. It also fails to exhibit the elevations of the two main peaks of the island of Taiwan, which are exactly 3886 and 3952 meters at their maximum elevation in northern and southern Taiwan, respectively. Also, it is interesting (a)

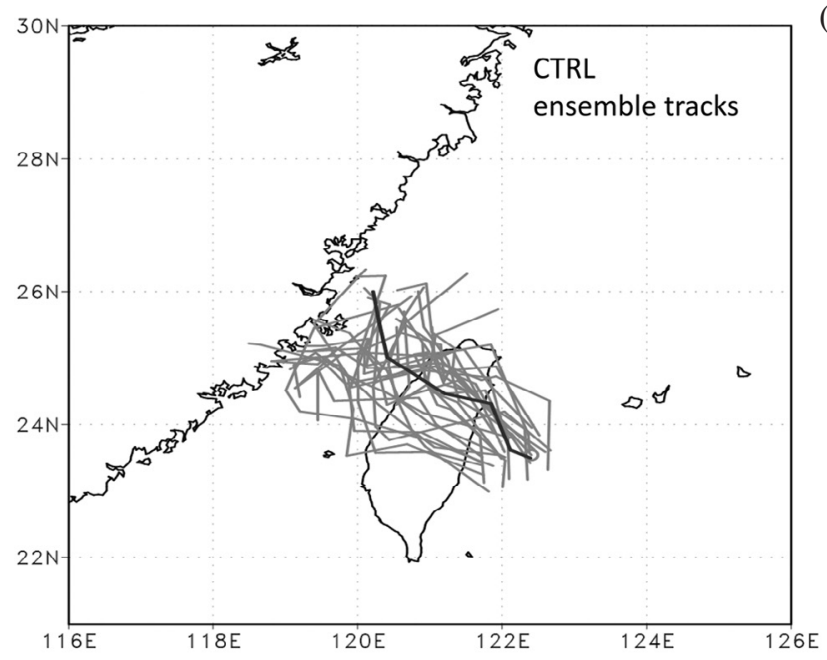

(b)

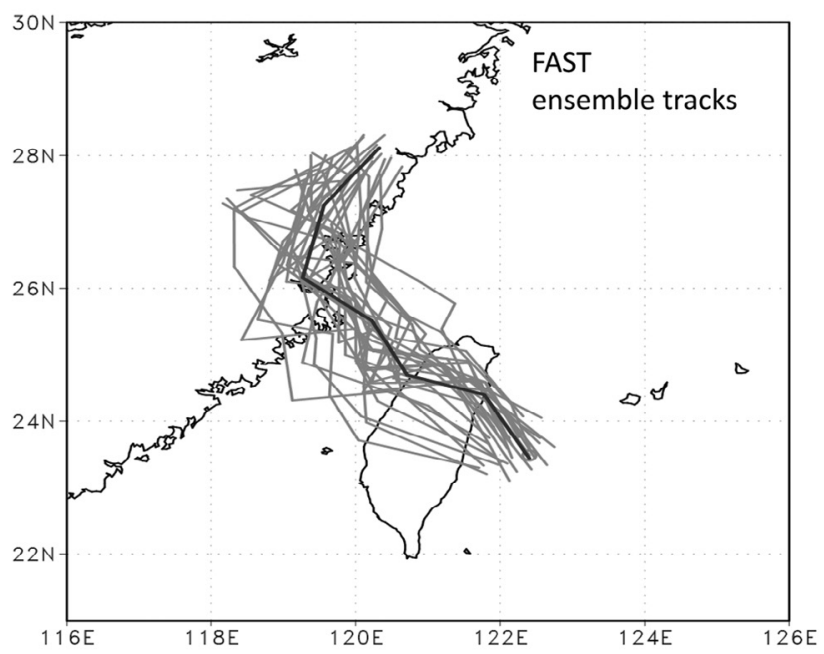

Fig. 7. The ensemble tracks (gray lines) of Morakot from 1200 UTC 07 August to 0000 UTC 09 August in (a) CTRL and (b) FAST. The ensemble mean tracks are shown in thick black line. 

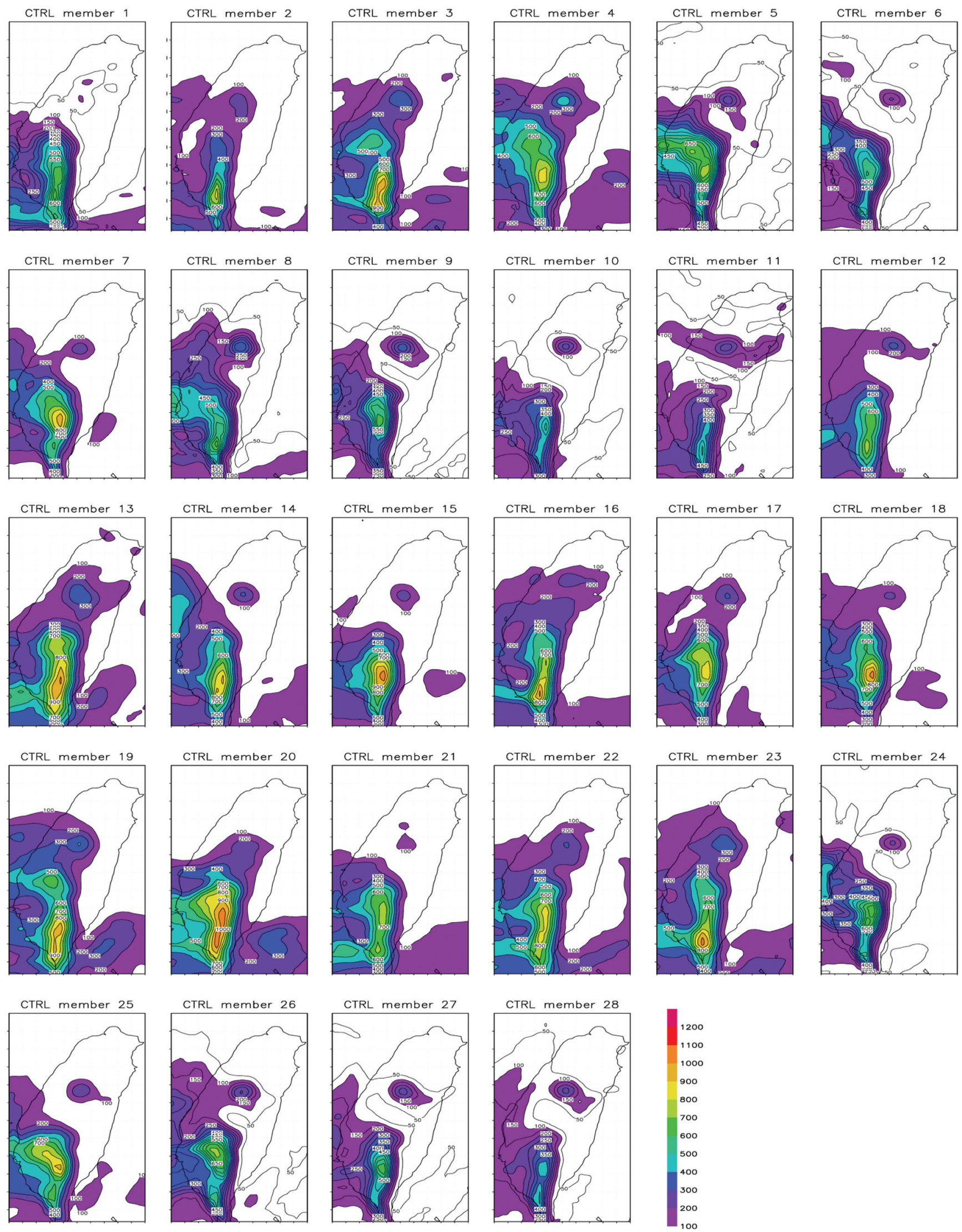

Fig. 8. The accumulated rainfall (mm) of 28 ensemble members in D2 from 1200 UTC 07 August to 1800 UTC 08 August in CTRL. 

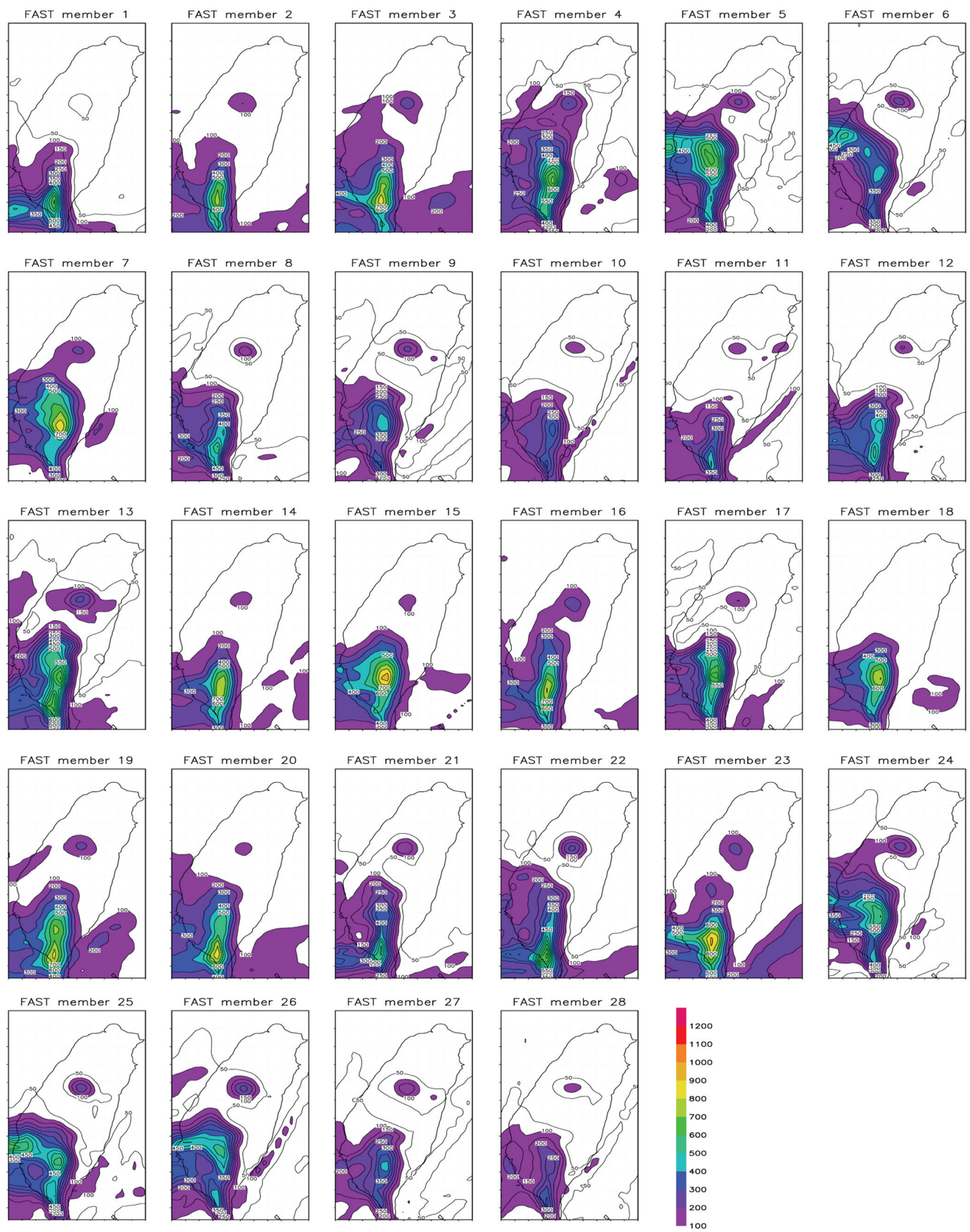

Fig. 9. Same as in Fig. 8, but for the FAST experiment from 1200 UTC 07 August to 0600 UTC 08 August. 


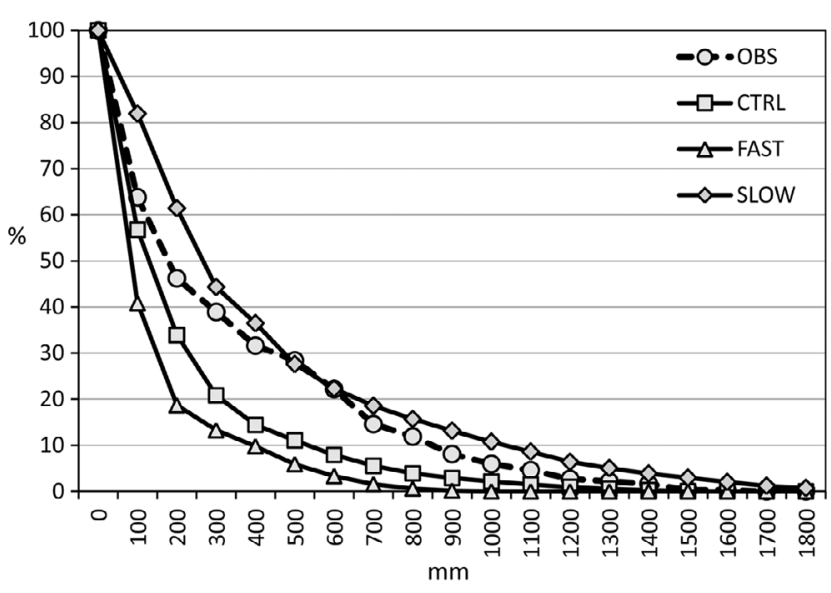

Fig. 10. The cumulative frequency of the total rainfall from 1200 UTC 07 August to 1800 UTC 08 August in CTRL (square marks), from 1200 UTC 07 August to 0600 UTC 08 August in FAST (triangle marks), and from 1200 UTC 07 August to 0000 UTC 10 August in SLOW (diamond marks) based on the data points over the island of Taiwan in domain 3. The observed cumulative frequency calculated from rain gauge observations in the same time interval as CTRL is also shown with dashed line and circle marks.

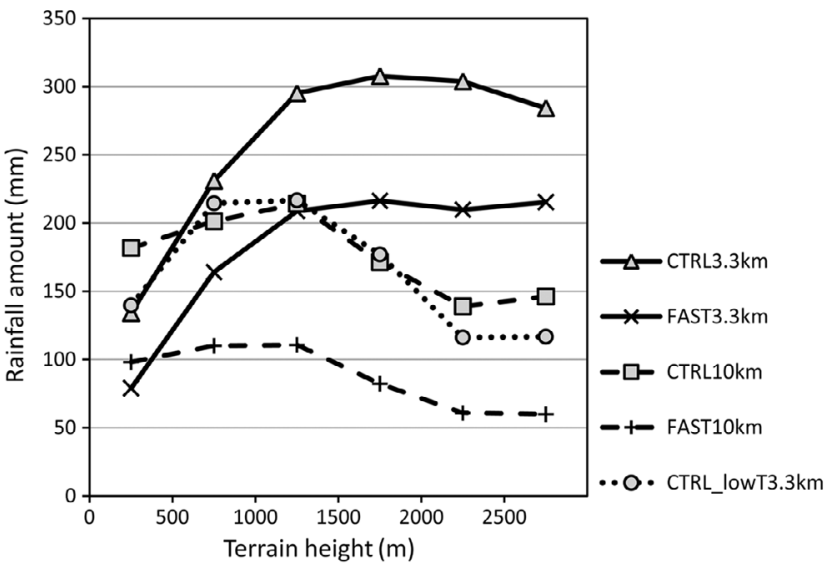

Fig. 11. The average accumulated rainfall amount of each group of data points over the island categorized based on their terrain height in D3 of CTRL (solid line with triangle markings), D3 of FAST (solid line with cross marks), D2 of CTRL (dashed line with square markings), and D2 of FAST (dashed line with plus signs), in addition to that in D3 of CTRL_lowT (dotted line with circle marks). The periods used to calculate the accumulated rainfall in each experiment are the same as in Fig. 10.
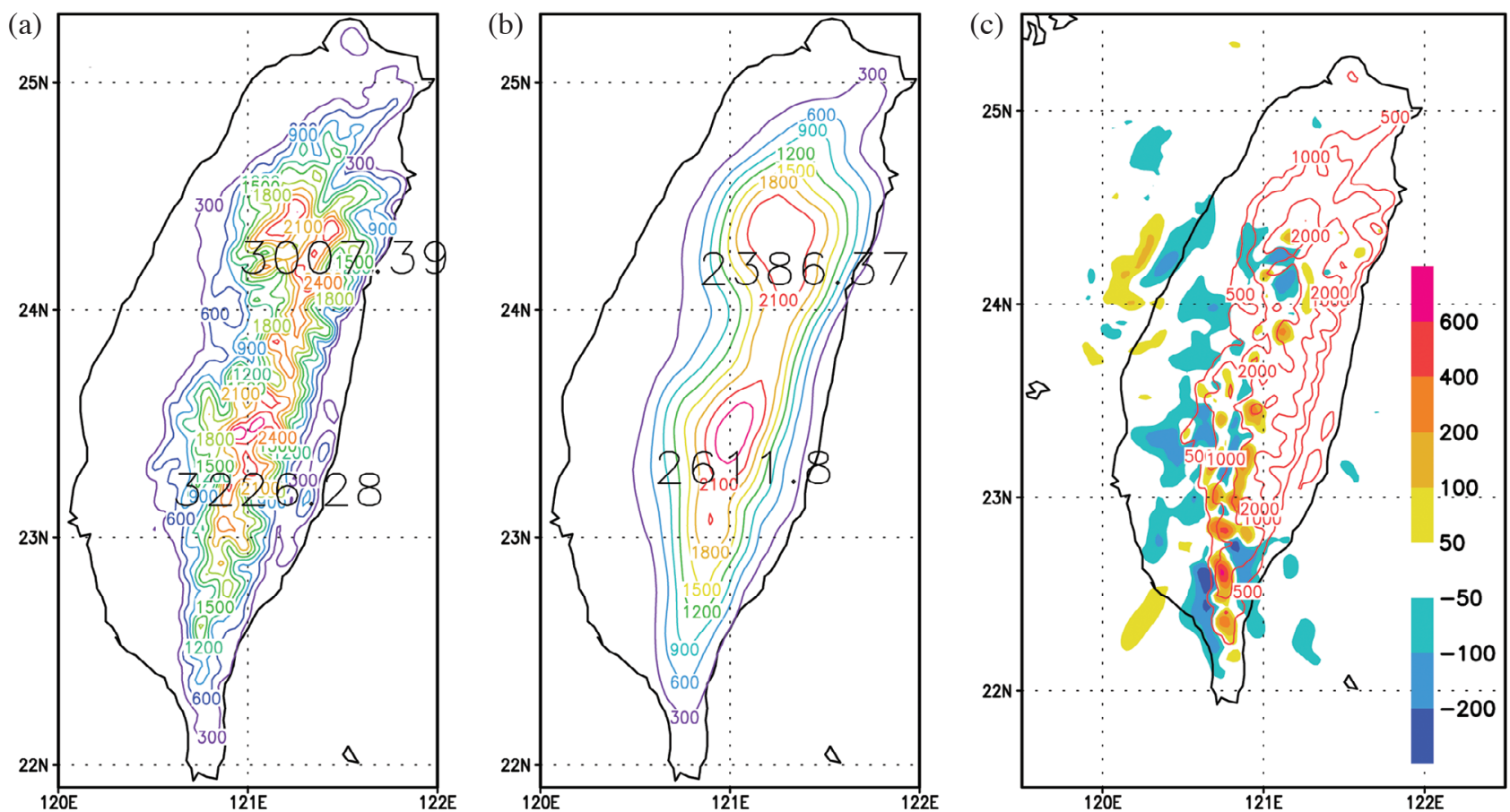

Fig. 12. Terrain height (m) used in the WRF model in (a) D3 with 3.33-km resolution and (b) D2 with 10-km resolution. The local maximum values of the two main peaks of the island of Taiwan are shown. (c) The difference in accumulated rainfall (mm) between CTRL and CTRL_lowT, superposed with the 3.33-km-resolution terrain height (red contour).

that the terrain height dependency of accumulated rainfall in 10-km-resolution domains is much different from that in 3.33-km-resolution domains. The average rainfall amount in areas with a lower terrain height is surprisingly larger than that in high mountain areas. A careful examination of the spatial distribution of accumulated rainfall in each domain (Figs. 6a, b and Figs. 8, 9) indicates that the rainfall amount in D2 is not only less than that in D3, but its pattern also diverts slightly to the southwest of that in D3, further toward the south-western plain of Taiwan and the Taiwan Strait. An 
additional experiment called CTRL_lowT is conducted to highlight the importance of terrain resolution. All settings in CTRL_lowT are the same as those in CTRL, except the model terrain of its D3, in which the terrain resolution is degraded to the same resolution as D2 $(10 \mathrm{~km})$, while the other model variables maintain the fine resolution of $3.33 \mathrm{~km}$. The overall terrain height dependency of accumulated rainfall in D3 of CTRL_lowT (dotted line in Fig. 11) is rather similar to that of D2 in CTRL (dashed line with square marks in Fig. 11), except that the increasing trend with terrain below 1000 meters is much closer to that in the $3.33-\mathrm{km}$-resolution domain (D3) of CTRL (solid line with triangle marks in Fig. 11). In addition, the comparison between the results from CTRL and CTRL_lowT is also made by directly subtracting the accumulated rainfall amount in CTRL_lowT from that in CTRL (Fig. 12c). The geographic shift in rainfall patterns can be clearly observed in Fig. 12c as the simulation with finer terrain resolution (CTRL) tends to produce more rainfall toward the mountain region than that with coarser terrain resolution. The increment can be up to $600 \mathrm{~mm}$ in some particular areas. In all, the terrain resolution can affect not only the accumulated rainfall amount associated with TCs, but also the rainfall distribution with respect to the terrain. The result from CTRL_lowT further emphasizes the importance of terrain resolution in numerical models to better simulate the topography-enhanced heavy precipitation as described in Wu et al. (2002).

\section{CONCLUDING REMARKS}

Typhoon Morakot formed on 3 August 2009 during a period with large-scale monsoon trough activity. It caused a catastrophic disaster in Taiwan mostly because of its record-breaking heavy precipitation. The maximum accumulated rainfall reached around $3000 \mathrm{~mm}$ within four days on the south-western slope of CMR. Morakot slowed down significantly from more than $20 \mathrm{~km} \mathrm{~h}^{-1}$ to around $10 \mathrm{~km} \mathrm{~h}^{-1}$ when it made landfall in Taiwan. The slow translation speed, together with the steep slope of the CMR in Taiwan, the prevailing south-westerly flow in the monsoon trough, and the presence of Typhoon Goni brought about this devastating weather event.

To quantitatively address the influence of the translation speed of Morakot on the rainfall amount over Taiwan, the method introduced by Wu et al. (2010b) is used to control the simulated TC tracks in the WRF model. Other than the observed track, the hypothetically accelerated and delayed tracks are constructed after 1200 UTC 07 August. Thus the center position and motion vector taken from these three tracks are directly assimilated into WRF model in the CTRL, FAST and SLOW experiments, respectively. The accumulated rainfall pattern during the landfall period of Morakot in CTRL generally matches the observations, with a maximum rainfall amount of $1800 \mathrm{~mm}$ located in the windward sloping areas in southern Taiwan, although the coverage of the low- to medium-rainfall area in the simulation is narrower than the observation. Regarding the FAST experiment, the $55 \%$ increase of the translation speed of simulated Morakot than that in CTRL leads to a 33\% reduction in the maximum accumulated rainfall within the similar rainfall patterns over Taiwan. In contrast, the accumulated rainfall amount increases remarkably in SLOW with a rather slow translation speed of the simulated TC. This simple study demonstrates the capability of the EnKF assimilation to examine some sensitivities related to TC movements by controlling TC translation speed directly.

On the basis of the ensemble data assimilation scheme, the 28 ensemble members can further provide additional information in terms of spread and other statistics. The accumulated rainfall amounts display large variance over the ensemble, considering the fact that the landfall locations of the simulated Morakot range from the northern-most tip of Taiwan to the main area of CMR. It highlights the necessity of ensemble simulations for the QPF. However, the dozens of the ensemble members also lead to a computational burden which limits the model resolution of the ensemble run. Therefore, only D1 and D2 can be used during the ensemble integration and data assimilation in this preliminary study. The $10-\mathrm{km}$ coarser resolution of D2 would lead to several issues and limitations in the analysis of the simulated rainfall amount as those discussed in the subsection $4 \mathrm{~d}$. For instance, the poor representation of Taiwan terrain with the insufficient terrain resolution will result in an underestimated accumulated rainfall amount with shifted pattern toward the low-elevation area. Therefore, more refined experiments with better initial ensemble and coherent resolutions are needed to overcome the deficiency in this study and further advance our understanding of this extreme rainfall event.

Acknowledgements This work is supported by the National Science Council of Taiwan under Grants NSC 98-2111-M002-008-MY3 and NSC 97-2111-M-002-016-MY3.

\section{REFERENCES}

Anderson, J., T. Hoar, K. Raeder, H. Liu, N. Collins, R. Torn, and A. Avellano, 2009: The data assimilation research testbed: A community facility. Bull. Amer. Meteorol. Soc., 90, 1283-1296, doi: 10.1175/2009BAMS2618.1. [Link]

Chen, C. S. and Y. L. Chen, 2003: The rainfall characteristics of Taiwan. Mon. Weather Rev., 131, 1323-1341, doi:10.1175/1520-0493(2003)131<1323:TRCOT>2.0. $\mathrm{CO} ; 2$. [Link]

Chen, Y. and C. Snyder, 2007: Assimilating vortex position with an ensemble Kalman filter. Mon. Weather Rev., 135, 1828-1845, doi: 10.1175/MWR3351.1. [Link] 
Dudhia, J., 1989: Numerical study of convection observed during the Winter Monsoon Experiment using a mesoscale two-dimensional model. J. Atmos. Sci., 46, 3077-3107, doi: 10.1175/1520-0469(1989)046<3077: NSOCOD $>2.0 . C O ; 2$. [Link]

Evensen, G., 1994: Sequential data assimilation with a nonlinear quasi-geostrophic model using Monte Carlo methods to forecast error statistics. J. Geophys. Res., 99, 10143-10162, doi: 10.1029/94JC00572. [Link]

Evensen, G., 2003: The ensemble Kalman filter: Theoretical formulation and practical implementation. Ocean Dyn., 53, 343-367, doi: 10.1007/s10236-003-0036-9. [Link]

Ge, X., T. Li, S.Zhang, and M. Peng, 2010: What causes the extremely heavy rainfall in Taiwan during Typhoon Morakot (2009)? Atmos. Sci. Lett., 11, 46-50, doi: 10. 1002/as1.255. [Link]

Grell, G. A. and D. Devenyi, 2002: A generalized approach to parameterizing convection combining ensemble and data assimilation techniques. Geophys. Res. Lett., 29, doi: 10.1029/2002GL015311. [Link]

Hendricks, E. A., J. R. Moskaitis, Y. Jin, R. M. Hodur, J. D. Doyle, and M. S. Peng, 2011: Prediction and diagnosis of Typhoon Morakot (2009) using the Naval Research Laboratory's mesoscale tropical cyclone model. Terr. Atmos. Ocean. Sci., 22, 579-594, doi: 10.3319/ TAO.2011.05.30.01(TM). [Link]

Hong, C. C., M. Y. Lee, H. H. Hsu, and J. L. Kuo, 2010: Role of submonthly disturbance and 40-50 day ISO on the extreme rainfall event associated with Typhoon Morakot (2009) in Southern Taiwan. Geophys. Res. Lett., 37, L08805, doi: 10.1029/2010GL042761. [Link]

Hong, S. Y. and J. O. J. Lim, 2006: The WRF SingleMoment 6-Class Microphysics Scheme (WSM6). J. Korean Meteor. Soc., 42, 129-151.

Hong, S. Y., J. Dudhia, and S. H. Chen, 2004: A revised approach to ice microphysical processes for the bulk parameterization of clouds and precipitation. Mon. Weather Rev., 132, 103-120, doi: 10.1175/1520-0493 (2004)132<0103:ARATIM>2.0.CO;2. [Link $]$

Hong, S. Y., Y. Noh, and J. Dudhia, 2006: A new vertical diffusion package with an explicit treatment of entrainment processes. Mon. Weather Rev., 134, 2318-2341, doi: 10.1175/MWR3199.1. [Link]

Huang, C. Y., C. S. Wong, and T. C. Yeh, 2011: Extreme rainfall mechanisms exhibited by Typhoon Morakot (2009). Terr. Atmos. Ocean. Sci., 22, 613-632, doi: 10.3319/TAO.2011.07.01.01(TM). [Link]

Jou, B. J.-D., Y. C. Yu, L. Feng, Y. M. Chen, C. S. Lee, and M. D. Cheng, 2010: Synoptic environment and rainfall characteristics of Typhoon Morakot (0908). Atmos. Sci., 38, 21-38. (in Chinese)

Lander, M. A., 1994: Description of a monsoon gyre and its effects on the tropical cyclones in the western North
Pacific during August 1991. Weather Forecast.,9, 640654, doi: 10.1175/1520-0434(1994)009<0640:DOAM $\mathrm{GA}>2.0 . \mathrm{CO} ; 2$. [Link]

Lander, M. A. and G. J. Holland, 1993: On the interaction of tropical-cyclone-scale vortices. I: Observations. $Q$. J. R. Meteorol. Soc., 119, 1347-1361, doi: 10.1002/qj. 49711951406. [Link]

Liang, J., L. Wu, X. Ge, and C. C. Wu, 2011: Monsoonal influence on Typhoon Morakot (2009). Part II: Numerical study. J. Atmos. Sci., 68, 2222-2235, doi: 10. 1175/2011JAS3731.1. [Link]

Lin, C. Y., H. M. Hsu, Y. F. Sheng, C. H. Kuo, and Y. A. Liou, 2010: Mesoscale processes for super heavy rainfall of Typhoon Morakot (2009) over Southern Taiwan. Atmos. Chem. Phys. Discuss., 10, 13495-13517. doi: 10.5194/acpd-10-13495-2010, 2010. [Link]

Lin, Y. L., S. Chiao, T. A. Wang, M. L. Kaplan, and R. P. Weglarz, 2001: Some common ingredients for heavy orographic rainfall. Weather Forecast., 16, 633-660, doi: 10.1175/1520-0434(2001)016<0633:SCIFHO>2. $0 . \mathrm{CO} ; 2 .[\underline{\mathrm{Link}}]$

Mlawer, E. J., S. J. Taubman, P. D. Brown, M. J. Iacono, and S. A. Clough, 1997: Radiative transfer for inhomogeneous atmospheres: RRTM, a validated correlated-k model for the longwave. J. Geophys. Res., 102, 1666316682, doi: 10.1029/97JD00237. [Link]

Nguyen, H. V. and Y. L. Chen, 2011: High-resolution initialization and simulations of Typhoon Morakot (2009). Mon. Weather Rev., 139, 1463-1491, doi: 10. 1175/2011MWR3505.1. [Link]

Snyder, C. and F. Zhang, 2003: Assimilation of simulated Doppler radar observations with an ensemble Kalman filter. Mon. Weather Rev., 131, 1663-1677, doi: 10.11 75//2555.1. [Link]

Szunyogh, I., E. J. Kostelich, G. Gyarmati, E. Kalnay, B. R. Hunt, E. Ott, E. Satterfield, and J. A. Yorke, 2008: A local ensemble transform Kalman filter data assimilation system for the NCEP global model. Tellus A, 60, 113-130, doi: 10.1111/j.1600-0870.2007.00274.x. [Link]

Tao, W. K., J. J. Shi, P. L. Lin, J. Chen, S. Lang, M. Y. Chang, M. J. Yang, C. C. Wu, C. Peters-Lidard, C. H. Sui, and B. J. D. Jou, 2011: High-resolution numerical simulation of the extreme rainfall associated with Typhoon Morakot. Part I: Comparing the impact of microphysics and PBL parameterizations with observations. Terr. Atmos. Ocean. Sci., 22, 673-696, doi: 10.3319/TAO.2011.08.26.01(TM). [Link]

Torn, R. D. and G. J. Hakim, 2008: Performance characteristics of a pseudo-operational ensemble Kalman filter. Mon. Weather Rev., 136, 3947-3963, doi: 10.1175/2008MWR2443.1. [Link]

Torn, R. D. and G. J. Hakim, 2009: Ensemble data assimilation applied to RAINEX observations of Hurricane 
Katrina (2005). Mon. Weather Rev., 137, 2817-2829, doi: 10.1175/2009MWR2656.1 [Link]

Wang, T.C.C., Y.S. Tang, C. H. Wei, P. L. Lin, Y.C.Liou, W. Y. Chang, C. B. Chou, B. T. Ji, and C. Y. Lin, 2010: The precipitation characteristics of Typhoon Morakot (2009) from radar analyses. Atmos. Sci., 38, 39-61. (in Chinese)

Whitaker, J. S. and T. M. Hamill, 2002: Ensemble data assimilation without perturbed observations. Mon. Weather Rev., 130, 1913-1924, doi: 10.1175/1520-0493(20 02)130<1913:EDAWPO > 2.0.CO;2. [Link]

Whitaker, J. S., T. M. Hamill, X. Wei, Y. Song, and Z. Toth, 2008: Ensemble data assimilation with the NCEP Global Forecast System. Mon. Weather Rev., 136, 463 -482, doi: 10.1175/2007MWR2018.1. [Link]

Willoughby, H. E., R. W. R. Darling, and M. E. Rahn, 2006: Parametric representation of the primary hurricane vortex. Part II: A new family of sectionally continuous profiles. Mon. Weather Rev., 134, 1102-1120, doi: 10. 1175/MWR3106.1. [Link]

Wu, C. C., 2001: Numerical simulation of Typhoon Gladys (1994) and its interaction with Taiwan terrain using the GFDL hurricane model. Mon. Weather Rev., 129, 1533 -1549, doi: 10.1175/1520-0493(2001)129<1533:NSO TGA>2.0.CO;2. [Link]

Wu, C. C. and Y. H. Kuo, 1999: Typhoons affecting Taiwan: Current understanding and future challenges. Bull. Amer. Meteorol. Soc., 80, 67-80, doi: 10.1175/ 1520-0477(1999)080<0067:TATCUA>2.0.CO;2. [Link]

Wu, C.C., T. H. Yen, Y.H. Kuo, and W. Wang, 2002: Rainfall simulation associated with Typhoon Herb (1996) near Taiwan. Part I: The topographic effect. Weather Forecast., 17, 1001-1015, doi: 10.1175/1520-0434(20 03)017<1001:RSAWTH $>2.0 . C O ; 2$. [Link]

Wu, C. C., T. S. Huang, W. P. Huang, and K. H. Chou, 2003: A new look at the binary interaction: Potential vorticity diagnosis of the unusual southward movement of Tropical Storm Bopha (2000) and its interaction with Supertyphoon Saomai (2000). Mon. Weather Rev., 131, 1289-1300, doi: 10.1175/1520-0493(2003) 131<1289:ANLATB>2.0.CO;2. [Link]
Wu, C. C., C. Y. Huang, M. J. Yang, F. C. Chien, J. S. Hong, and T. H. Yen, 2010a: Typhoon Morakot (2009) and a special review on the current status and future challenge of tropical cyclone simulation. Atmos. Sci., 38, 99-134. (in Chinese)

Wu, C. C., G. Y. Lien, J. H. Chen, and F. Zhang, 2010b: Assimilation of tropical cyclone track and structure based on the Ensemble Kalman Filter (EnKF). J. Atmos. Sci., 67, 3806-3822, doi: 10.1175/2010JAS3444.1. [Link]

Wu, C. C., Y. H. Huang, and G. Y. Lien, 2011a: Concentric eyewall formation in Typhoon Sinlaku (2008) - Part I: Assimilation of T-PARC data based on the ensemble Kalman filter (EnKF). Mon. Weather Rev., accepted.

Wu, L., J. Liang, and C. C. Wu, 2011b: Monsoonal influence on Typhoon Morakot (2009). Part I: Observational analysis. J. Atmos. Sci., 68, 2208-2221, doi: 10. 1175/2011JAS3730.1. [Link]

Yeh, T. C., H. C. Kuo, K. C. Lu, S. C. Wang, and Y. L. Chen, 2010: Verification on Typhoon Morakot track and rainfall forecast issued by the Central Weather Bureau. Atmos. Sci., 38, 85-98. (in Chinese)

Zhang, F., C. Snyder, and J. Sun, 2004: Impacts of initial estimate and observation availability on convectivescale data assimilation with an ensemble Kalman filter. Mon. Weather Rev., 132, 1238-1253, doi: 10.1175/152 0-0493(2004)132<1238:IOIEAO>2.0.CO;2. [Link]

Zhang, F., Z. Meng, and A. Aksoy, 2006: Tests of an ensemble Kalman filter for mesoscale and regional-scale data assimilation. Part I: perfect model experiments. Mon. Weather Rev., 134, 722-736, doi: 10.1175/MWR 3101.1. [Link]

Zhang, F., Y. Weng, J. A. Sippel, Z. Meng, and C. H. Bishop, 2009: Cloud-resolving hurricane initialization and prediction through assimilation of Doppler radar observations with an ensemble Kalman filter. Mon. Weather Rev., 137, 2105-2125, doi: 10.1175/2009MWR2645.1. [Link]

Zhang, F., Y. Weng, Y. H. Kuo, J. S. Whitaker, and B. Xie, 2010: Predicting Typhoon Morakot's catastrophic rainfall with a convection-permitting mesoscale ensemble system. Weather Forecast., 25, 1816-1825, doi: 10.1175/2010WAF2222414.1. [Link] 